\title{
A Tradeoff between Rich Multipath and High Receive Power in MIMO Capacity
}

\author{
Zimu Cheng, Binghao Chen, and Zhangdui Zhong \\ State Key Laboratory of Rail Traffic Control and Safety, Beijing Jiaotong University, Beijing 100044, China \\ Correspondence should be addressed to Zimu Cheng; 10120073@bjtu.edu.cn
}

Received 27 July 2012; Accepted 24 November 2012

Academic Editor: Ai Bo

Copyright (C) 2013 Zimu Cheng et al. This is an open access article distributed under the Creative Commons Attribution License, which permits unrestricted use, distribution, and reproduction in any medium, provided the original work is properly cited.

\begin{abstract}
A discussion about which of the two factors, rich multipath (in NLOS) or signal-to-noise ratio (SNR) (usually in LOS), affects the Multiple-Input Multiple-Output (MIMO) channel capacity more is presented in this paper. This interesting discussion is investigated by performing simulations using simple circle scatterer model and WINNER II channel model. And the simulation shows that these two factors behave differently as the channel condition varyies. When the scatterer number in channel is low, the high receive SNR is more important to capacity. The multipath richness will have greater influence when the scatterer number exceeds a certain threshold. However, the channel capacity will not change much as the scatterers continue to increase.
\end{abstract}

\section{Introduction}

Multiple-Input Multiple-Output (MIMO) is a hot research topic that has always attracted much attention in recent decades. When multiple antennas are deployed at both transmit side and receive side, the performance of communication system can be enhanced significantly.

The MIMO performance highly depends on the propagation environments and channel model structures. Two channel conditions, that is, NLOS (Non-line-of-sight) and LOS (Line-of-sight), are commonly used in propagation research. In general, if a strong LOS path exists in environment, it is called the Ricean channel. If there is no LOS path, the receive signal will follow Rayleigh distribution. In Rayleigh assumption, the multipath is scattered by the rich scatterers uniformly distributed around the receiver. And the multipath richness is important for MIMO system, for it will provide a large number of eigenvalues of MIMO channel. While in LOS scenario, generally the LOS path is stronger than scattering components and leads to a high receive SNR which will also contribute to MIMO capacity. But a high SNR associated with LOS often implies a low degree of scattering which, however, will cause the capacity loss again. This paper is going to talk about whether the rich multipath or high receive SNR is more important to MIMO capacity.
The discussions about which of these two factors is more important have started in the literature. Wallace and Jensen discuss the MIMO capacity variation with SNR and multipath richness using full-wave indoor finite-difference time domain (FDTD) simulations [1]. The relationship of SNR, effective degrees of freedom (EDOF), and capacity is studied. Also a slight change of multipath richness is observed only in the indoor environment. Malik studies MIMO capacity and multipath scaling in ultrawideband (UWB) channels [2]. It is found that in a rich scattering environment, the indoor UWB system capacity can be dramatically increased by using MIMO array. Matthaiou et al. investigates the impact of sparse multipath on MIMO channel performance [3]. According to the indoor measurement data, it shows that physical nature of scattering environment has a noticeable impact on the degree of freedom afforded by a sparse multipath MIMO channel. The ergodic capacity has strong dependency on the sparse multipath environment. Koch and Lapidoth research the high-SNR behavior of the capacity of noncoherent multipath fading channels [4]. At high SNR, if the number of path is finite, the capacity grows to double logarithmically with the SNR. Meanwhile, the high-SNR behavior of the capacity of multipath fading channels depends critically on the assumed channel model. A slight change in the channel model might lead to completely different capacity 
results. Saad et al. focus on the relationship between channel correlation, eigenvalues, and capacity [5]. They draw the conclusion that the eigenmodes of channel are independent of the SNR, but depend on channel correlation. And the maximum eigenmode of MIMO channel increases with the channel correlation while all other eigenmodes decrease. Knopp et al. investigate the impact of sparse multipath components on the LOS MIMO channel capacity using inroom measurements [6]. It is found that single but strong deterministic multipath signal distinctly enhances the channel capacity in low-rank LOS channel, while no significant capacity change occurs in high-rank LOS channel due to the reflected waves. However, most of the measurements above are taken indoors and the corresponding simulations and theoretical analysis are considered in the indoor environment only. More general channel models are used in this paper, and the relationship between the number of scatterers and eigenvalues is analyzed.

To avoid the Rayleigh channel model limitation that the scatterers' distribution is ideal and does not agree well with the real case, the circle scatterer model and WINNER II channel models are used in this paper, for they can be manipulated flexibly and are much closer to the real case that the multipath is a kind of sparse in real channel. Although a general geometry-based stochastic model, consisting of tworing and ellipse scatterers with single-bounce and doublebounce paths, is proposed in [7-9], the circle scatterer model and WINNER II model used in this paper can also cover most of the scenarios. More importantly, it is easy to change the number of scatterers or clusters in these two models, which will make us concentrate on the relationship between scatterer number and channel capacity. Besides the discussion, the effect of scatterer number on MIMO channel structure, that is, the eigenvalues for all subchannels, is analyzed as well. The discussion is based on mobile scenario. For the high velocity of receiver, the channel information is unknown at the transmitter. So equal power allocation strategy is applied, which will be described in details later.

The paper is organized as follows. In Section 2, circle scatterer model and WINNER II channel model are introduced. MIMO channel capacity will be derived and developed in Section 3. Section 4 is devoted to the simulation result and the conclusions are drawn in Section 5.

\section{MIMO Channel Model}

2.1. Circle Scatterer Model. This model is aimed at generating multiple-point scatterers around the receiver. The scatterers can be placed in a circle with the receiver in the center. The receive signal consists of the waves scattered once by each point scatterer. The basic simulation idea is to calculate the complex envelop for each possible combination of transmit and receive antennas, like what Figure 1 shows. Then, the ensemble of complex envelop constitutes the channel transmission matrix. From there, singular value decomposition (SVD) of $H$ is performed for assessing the available capacity [10].

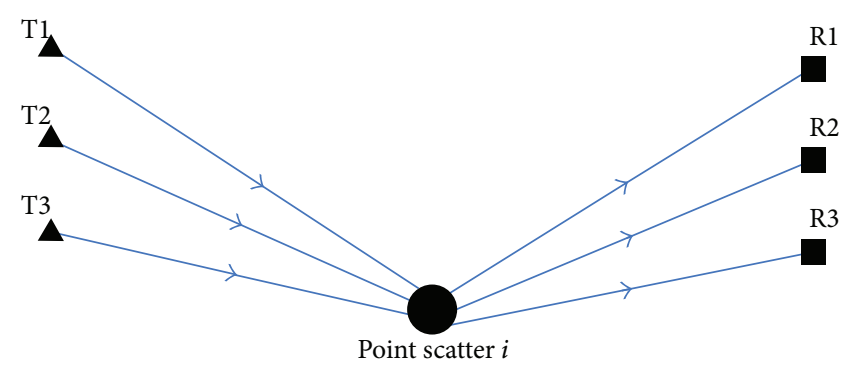

Figure 1: Circle scatterer model.

The general representation of the complex envelop received at antenna $j$ from transmit antenna $i$ is given by

$$
r^{i j}=\sum_{n=1}^{N} a_{n} \exp \left(-j k_{c} d_{n}^{i j}+j \Phi_{n}^{i j}\right),
$$

where coefficients $i j$ indicate Tx-Rx antenna pair, $n$ indicates the scatterer number, $N$ indicates the total number of scatterers (paths), and $a_{n}$ indicates the transmit power of the path, while $d_{n}^{i j}$ and $\Phi_{n}^{i j}$ are the distance of Tx-scatterer-Rx and the phase of the received wave, respectively. $k_{c}$ is the propagation constant.

2.2. WINNER II Channel Model. The WINNER II channel model is a geometry-based stochastic channel model, which can generate an arbitrary MIMO channel matrix for defined scenarios. It is ray-based double directional multilink and antenna-independent model for MIMO systems. The statistical distributions of channel parameters such as AoA (Angle of Arrival), DoA (Angle of Departure), delay spread, and delay values are obtained from channel measurements. In the simulation, the parameters are determined stochastically from the distributions for each channel sample. Fixed 20 rays compose a cluster, which is considered as a propagation path diffused in space domain. The channel impulse response coefficients are generated by combining contributions of all rays which are characterized by small scale parameters.

One single link of the WINNER II channel model is illustrated in Figure 2 [11], in which the parameters for generation of channel matrix are also illustrated. Each circle with several dots represents scattering region causing one cluster. The number of clusters varies from 8 to 20 depending on different scenarios.

$\varphi$ and $\phi$ are the AoA and AoD, respectively, $\tau$ is the cluster delay, and $\sigma$ is the delay spread. $\bar{v}$ is the speed of MS. So the channel matrix of WINNER II MIMO channel model is given by [11]

$$
H(t ; \tau)=\sum_{n=1}^{N} H_{n}(t ; \tau)
$$

where

$$
H_{n}(t ; \tau)=\iint F_{r x}(\varphi) h_{n}(t ; \tau, \phi, \varphi) F_{t x}^{T}(\phi) d \phi d \varphi,
$$

where $N$ is the total number of paths, and $F_{t x}$ and $F_{r x}$ refer to antenna array response matrices for the transmitter and 


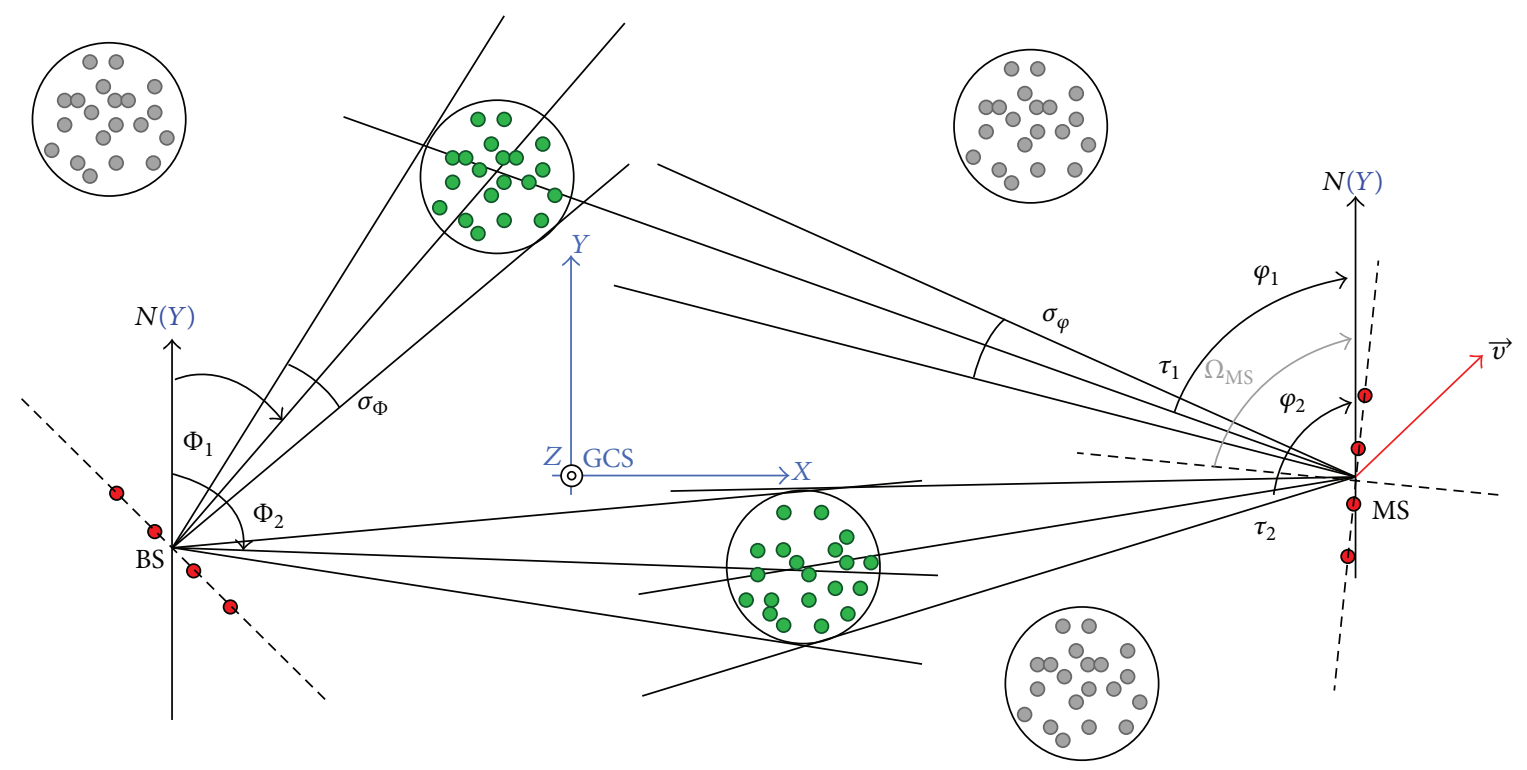

FIGURE 2: WINNER II channel model.

receiver, respectively. $h_{n}$ is the propagation channel response matrix for cluster $n$.

The channel response for cluster $n$ from transmitter antenna elements $s$ to receiver transmitter elements $u$ is given by [11]

$$
\begin{aligned}
H_{u, s, n}(t ; \tau) & \\
= & \sum_{m=1}^{M}\left[\begin{array}{l}
F_{r x, u, V}\left(\varphi_{n, m}\right) \\
F_{r x, u, H}\left(\varphi_{n, m}\right)
\end{array}\right]^{T}\left[\begin{array}{ll}
\alpha_{n, m, V V} & \alpha_{n, m, V H} \\
\alpha_{n, m, H V} & \alpha_{n, m, H H}
\end{array}\right] \\
& \times\left[\begin{array}{l}
F_{t x, s, V}\left(\phi_{n, m}\right) \\
F_{t x, s, H}\left(\phi_{n, m}\right)
\end{array}\right] \\
& \times \exp \left(j 2 \pi \lambda_{0}^{-1}\left(\bar{\varphi}_{n, m} \cdot \bar{r}_{r x, u}\right)\right) \\
& \times \exp \left(j 2 \pi \lambda_{0}^{-1}\left(\bar{\phi}_{n, m} \cdot \bar{r}_{t x, s}\right)\right) \\
& \times \exp \left(j 2 \pi v_{n, m} t\right) \delta\left(\tau-\tau_{n, m}\right)
\end{aligned}
$$

where $F_{t x, u, H}$ and $F_{r x, u, V}$ represent the antenna element $u$ field patterns for horizontal and vertical polarizations. $\alpha_{n, m, H H}$ and $\alpha_{n, m, H V}$ represent the complex gains of horizontalto-horizontal and horizontal-to-vertical polarization of ray $n, m$. And $\lambda_{0}$ is the wave length of carrier frequency. Meanwhile, $\bar{\varphi}_{n, m}$ is AoA unit vector, and $\bar{\phi}_{n, m}$ is AoD unit vector. $\bar{r}_{r x, u}$ and $\bar{r}_{t x, s}$ represent the location vector of elements $u$ and $s$ respectively. $v_{n, m}$ refers to the Doppler shift component of ray $n, m$. And the superscript $(\cdot)^{T}$ means transposition. All the small scale parameters mentioned above are time variant for the reason that the radio channel is modeled dynamically. More details about parameters generation in WINNER II model can be found in [11].

\section{MIMO Channel Capacity}

A MIMO system model can be expressed as

$$
y=H x+n
$$

where $x$ and $y$ are the vectors of transmitted and received signals at one sample time, respectively, and $n$ is the addictive white Gaussian noise (AWGN). If it is considered that the transmitter has $n_{t}$ antennas while the receiver has $n_{r}$ antennas, then $H$ should be an $n_{r} \times n_{t}$ matrix.

According to the information theory, the ergodic capacity is given by [12]

$$
C=\mathrm{E}\left[\log \operatorname{det}\left(I_{n_{r}}+\frac{\mathrm{SNR}}{n_{t}} H H^{*}\right)\right] \text {, }
$$

where $I_{n_{r}}$ is an $n_{r}$ rank unit matrix and SNR $=P / N_{0}$ is the signal-to-noise ratio at receiver.

The channel also can be divided into several parallel independent subchannels by the method of SVD. The number of subchannels is the same as the number of singular values and the gains of these subchannels are related to the value of the singular values. Hence, the total channel capacity can be obtained by summing the capacities of all the subchannels. In that case, the ergodic capacity can be expressed as follows [12]:

$$
C=\sum_{i=1}^{n_{\min }} \log \left(1+\frac{\mathrm{SNR}}{n_{t}} \lambda_{i}^{2}\right),
$$


where $n_{\min }=\min \left\{n_{t}, n_{r}\right\}, \lambda_{i}$ are singular values of channel matrix. For the reason that $\lambda_{i}$ is nonnegative, the equation above can be concluded as

$$
\begin{aligned}
C & =\log \prod_{i=1}^{n_{\min }}\left(1+\frac{\mathrm{SNR}}{n_{t}} \lambda_{i}^{2}\right) \\
& \leq \log \left(\frac{\sum_{i=1}^{n_{\min }}\left(1+\left(\mathrm{SNR} / n_{t}\right) \lambda_{i}^{2}\right)}{n_{\min }}\right)^{2} \\
& =2 \log \left(\frac{\sum_{i=1}^{n_{\min }}\left(1+\left(\mathrm{SNR} / n_{t}\right) \lambda_{i}^{2}\right)}{n_{\min }}\right) \\
& =2 \log \left(1+\frac{\mathrm{SNR}}{n_{t} n_{\min }} \sum_{i=1}^{n_{\min }} \lambda_{i}^{2}\right) .
\end{aligned}
$$

If the sum of $\lambda_{i}$ is a constant number, then the MIMO channel capacity could reach the maximum in a given scenario when all $\lambda_{i}$ are equal.

The power allocation strategy also affects the capacities of the subchannels. There are two strategies in common use, waterfilling algorithm and equal power allocation scheme [12]. A lot of researches adopt waterfilling algorithm since they assume that the transmitter learns the channel state information (CSI) before it transmits the data vector. However, there are some environments that the transmitter could not obtain the CSI, like in high speed moving timevariant scenario. Hence, in this paper, equal power allocation strategy is used in these particular situations.

\section{Simulation Result}

In this section, based on the formula described above, the performance of MIMO capacity with different scatterer number, that is, different multipath richness, is investigated in simulations. Meanwhile, the effect of scatterer number on MIMO channel structure is shown as well.

4.1. Circle Scatterer Model. The simulation of this model is depicted in Figure 3. The BS is located $500 \mathrm{~m}$ away from the MS. The velocity of the MS is $10 \mathrm{~m} / \mathrm{s}$. The scatterers are placed randomly on the circle around MS with the radius of $200 \mathrm{~m}$. The carrier frequency is $900 \mathrm{MHz}$ and the wavelength is $0.33 \mathrm{~m}$. The total transmit power is set to $0 \mathrm{dBm}$ and SNR is set to $20 \mathrm{~dB}$ as well. $5 \times 5$ antennas are used and they are separated by one wavelength at each side. A direct link between BS and MS is considered as the LOS component in this scenario. The simulation has run 400 times to get a mean value at each data point. In the simulation, the power of all scattering components is almost the same. And the Ricean $K$ factor is introduced to control the LOS path energy.

The effects of NLOS and LOS with different Ricean $K$ factors on ergodic channel capacity changing with scatterer number are given in Figure 4. If there are more than 5 scatterers, it can be noticed that the NLOS case provides more capacity than the LOS case with the same number of scatterers. In order to accomplish the same capacity as NLOS case, more scatterers are needed in LOS scenario. For

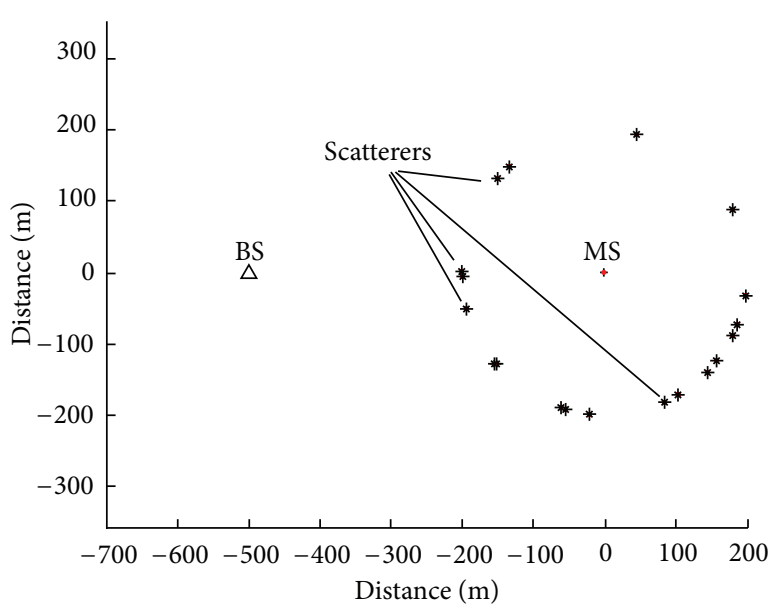

FIgure 3: Circle scatterer model setup.

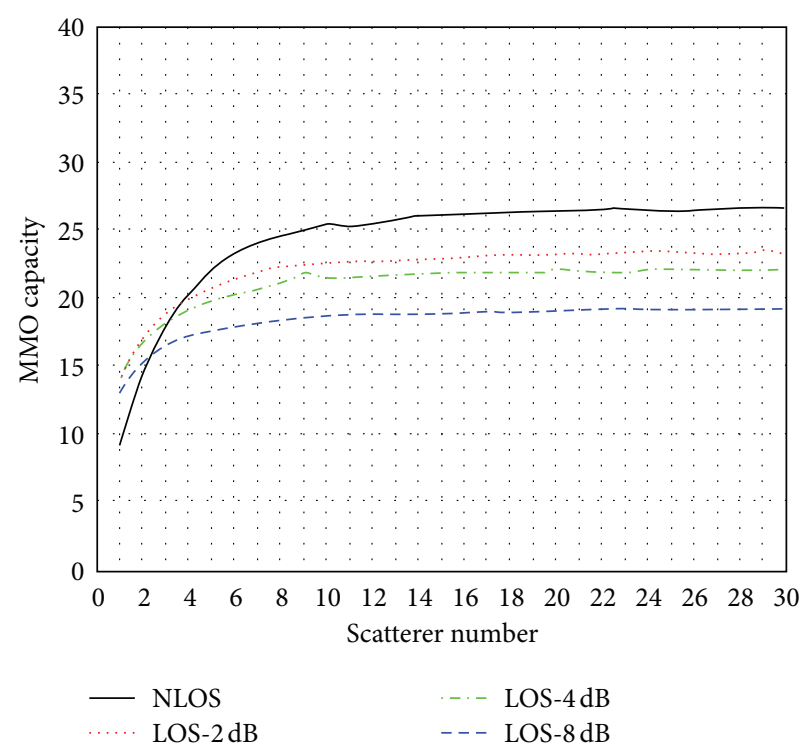

Figure 4: MIMO capacity comparison.

example, when the channel capacity is around $23 \mathrm{bit} / \mathrm{s} / \mathrm{Hz}$, the scatterer number for NLOS scenario is about 5 , while 5 more scatterers, that is, about 10 scatterers in total, are required in LOS scenario. When the scatterers are less than 5 , the enhanced receive power brought by LOS path is more important to capacity. And among those three LOS cases, the smaller the Ricean $K$ factor is, the better performance the system could offer. Meanwhile, when the number of scatterers reaches 25 , the system is already in rich multipath environment. In this situation, there is a little increase in capacity no matter how many scatterers are added.

The reason of MIMO capacity change is that multipath richness and LOS power (also Ricean $K$ factor) will affect the eigenvalues distribution. Figures 5 and 6 show the change of eigenvalues with the scatterer number in NLOS and LOS$8 \mathrm{~dB}$ scenarios, respectively. In NLOS scenario, the first eigenvalue $\lambda_{1}$ is quite big and the others are quite small 


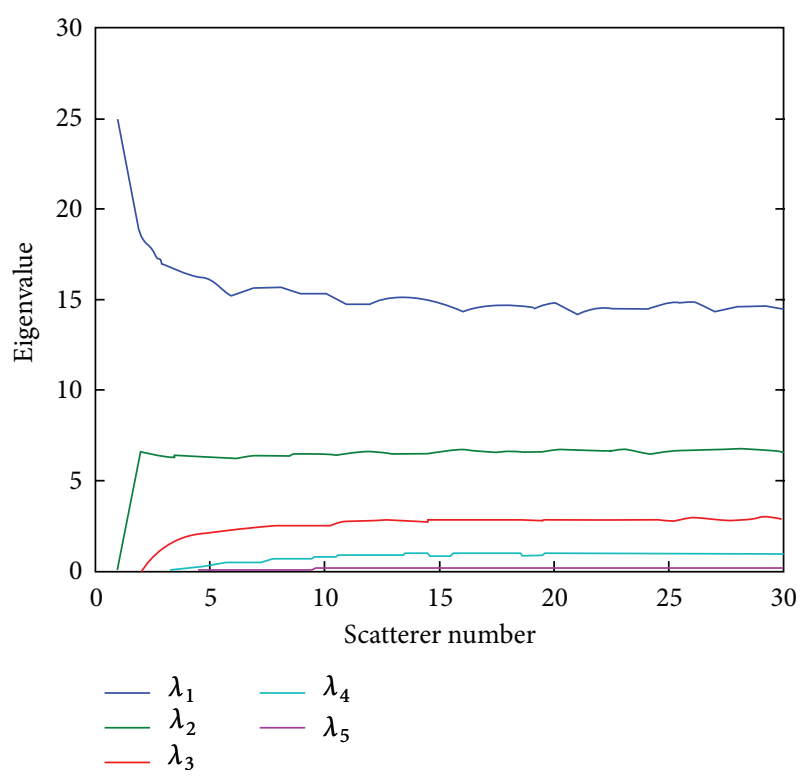

FIGURE 5: Eigenvalues comparison in NLOS.

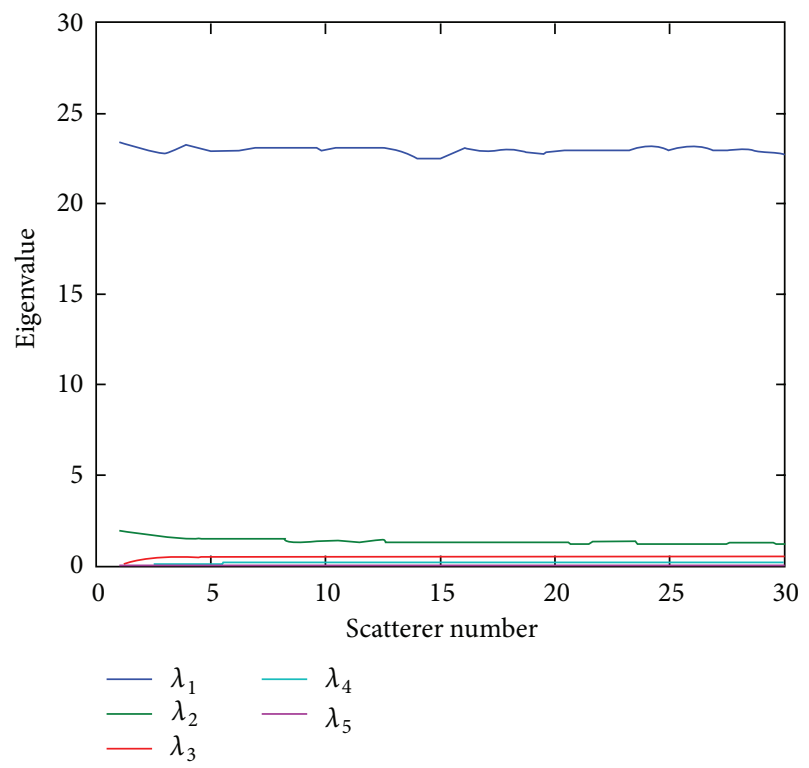

FIGURE 6: Eigenvalues comparison in LOS.

initially. However, with the increase of the scatterer number, $\lambda_{1}$ decreases to a lower level, while the others increase at the same time. More scatterers are helpful in NLOS scenario to reduce the gap between different eigenvalues, which is much closer to the ideal case given by expression (8). However, this case only happens in a certain scatterer number range. When the scatterer number is from 2 to 8 , the eigenvalues of all subchannels vary significantly. It shows that the eigenvalues will converge in NLOS scenario as the scatterers increase. Then, the scatterer number will not affect the MIMO channel structure much. According to Figure 6, it can be seen that, due to the LOS component, $\lambda_{1}$ always stays at a high level. $\lambda_{2}$ to $\lambda_{5}$ are so small that the channel matrix could not achieve full-rank. It seems that the eigenvalue $\lambda_{1}$ is much higher than the other eigenvalues. In order to increase the third or fourth eigenvalue, adding scatterers only works in a short range and the effect is limited.

4.2. WINNER II Channel Model. D2 scenario (rural moving networks) is used in the simulation. It represents radio propagation in environments where both the access point and the receiver are moving at very high speed in a rural area. A typical example of this scenario occurs in carriages of high-speed trains where wireless coverage is provided by the so-called moving relay stations (MRSs). Hence, There are two parts in D2 scenario: D2a (BS-MRS) and D2b (MRSMS). WINNER II defines D2a as LOS case which has 8 clusters. The parameters in D2b scenario are not defined in WINNER II but it recommends using the parameters in D1 scenario (rural macrocell) instead. So, D2b is typically seen as NLOS case with 10 clusters. In the simulation, the distance between BS and MS is $500 \mathrm{~m}$, and the height of BS and MS is $32 \mathrm{~m}$ and $1.5 \mathrm{~m}$, respectively. The velocity of MS is limited at $55.56 \mathrm{~m} / \mathrm{s}$ in LOS and $10 \mathrm{~m} / \mathrm{s}$ in NLOS. The carrier frequency is $5.25 \mathrm{GHz}$ and the wavelength is $0.057 \mathrm{~m}$. The SNR is set to $20 \mathrm{~dB}$ as well. $4 \times 4$ antennas are used and they are separated by $1 \mathrm{~cm}$ at each side. In this scenario, Ricean $K$ factor in LOS situation is $7 \mathrm{~dB} .100$ samples are taken in one simulation, and 500-time simulations are run for one cluster number.

Figure 7 shows the MIMO capacity of LOS and NLOS in D2 scenario. It can be seen that at the beginning, the capacity is increasing for the reason that the clusters could offer some DOF. However, the capacity has a slight decrease when the cluster number continues increasing. This is because WINNER II normalizes the total power to one and allocates the cluster power according to their delays. When the number of cluster increases, the power allocated to each cluster would decline. Some of the clusters get quite small power that the signal even could not reach the receiver through the channel fading. In that case, the total power received gets smaller. Meanwhile, when there is only one cluster, LOS case has an advantage. When the number is 2, the capacity of NLOS and LOS are almost equal. Then, the capacity of NLOS becomes bigger. But it is also shown that the influence of the capacity decrease affects NLOS more. That is because in LOS case, most of the power would allocate to the LOS component; multipath is not the crucial factor.

Figures 8 and 9 illustrate the eigenvalues in LOS and NLOS situation, respectively. There is little change during the cluster number increasing. Comparing these two cases, $\lambda_{1}$ is a little higher in LOS than in NLOS scenario, while in NLOS, the second eigenvalue $\lambda_{2}$ has a bigger value.

Hence, in this WINNER channel model simulation, the path number (cluster number) does not affect the capacity as much as the former model.

\section{Conclusion}

In this paper, the effect of rich multipath and high receive power on MIMO channel capacity is investigated. A simple circle scatterer model and WINNER II channel model are used to simulate the channel matrix. From the simulation 


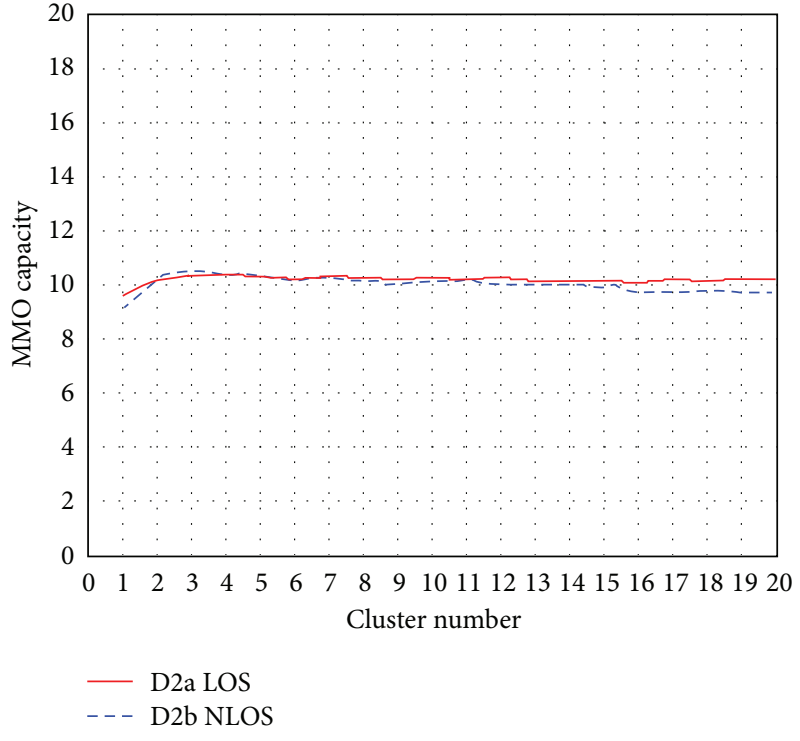

FIGURE 7: MIMO capacity comparison.

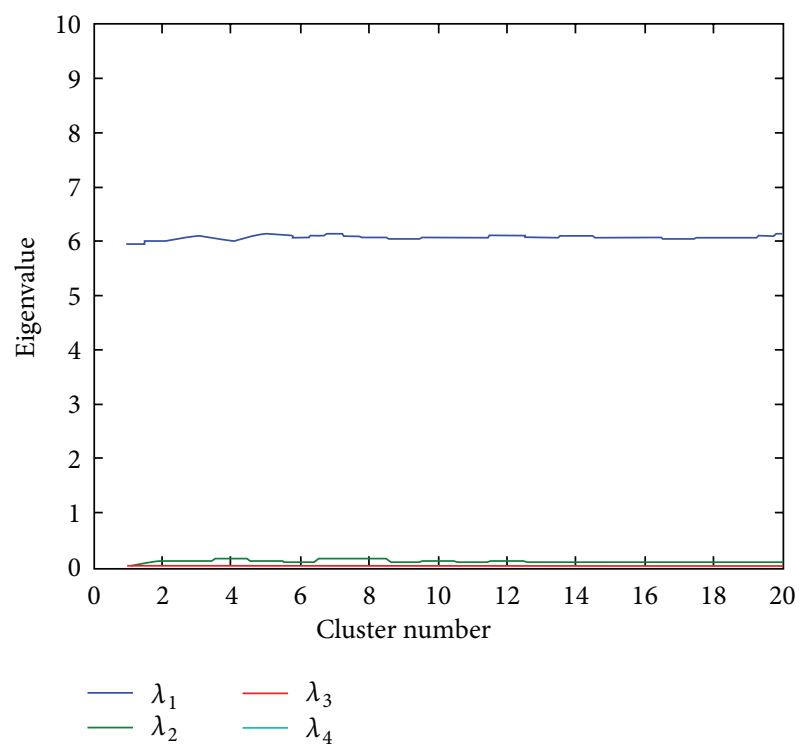

FIGURE 8: Eigenvalues comparison in LOS.

results, it can be seen when the scatterer number exceeds a certain threshold, the multipath richness prevails over the high receive SNR. When the scatterers are few, the high receive SNR is more important. However, the multipath richness will have little influence on capacity as the scatterers continue to increase.

\section{Acknowledgments}

The project is supported by the Program for New Century Excellent Talents under Grant NCET-09-0206, the National Natural Science Foundation of China under Grant 60830001, the Key Project of State Key Laboratory of Rail Traffic Control and Safety under Grants RCS2008ZZ006

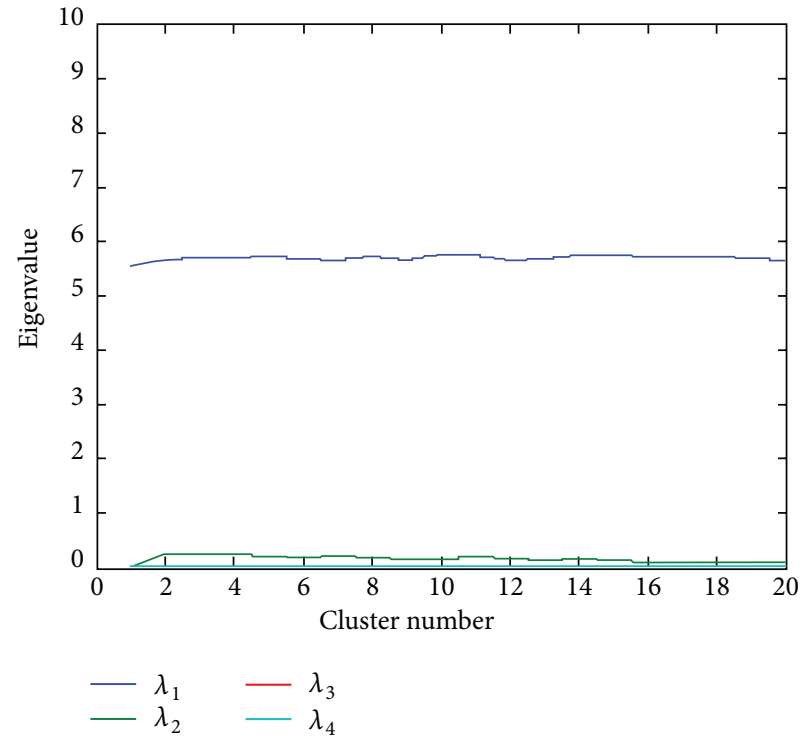

Figure 9: Eigenvalues comparison in NLOS.

and RCS2011ZZ008, Program for Changjiang Scholars and Innovative Research Team under Grant no. IRT0949, the Project of State Key Laboratory of Rail Traffic Control and Safety under Grants RCS2008ZT005 and RCS2010ZT012, Beijing Natural Science Foundation (4112048), and "the Fundamental Research Funds for the Central Universities" under Grants nos. 2010JBZ008 and 2011YJS010.

\section{References}

[1] J. W. Wallace and M. A. Jensen, "MIMO capacity variation with SNR and multipath richness from full-wave indoor FDTD simulations," in Proceedings of the IEEE International Antennas and Propagation Symposium and USNC/CNC/URSI North American Radio Science Meeting, vol. 2, pp. 523-526, June 2003.

[2] W. Q. Malik, "MIMO capacity and multipath scaling in ultrawideband channels," Electronics Letters, vol. 44, no. 6, pp. 427-428, 2008.

[3] M. Matthaiou, A. M. Sayeed, and J. A. Nossek, "Sparse multipath MIMO channels: performance implications based on measurement data," in Proceedings of the IEEE 10th Workshop on Signal Processing Advances in Wireless Communications (SPAWC '09), pp. 364-368, June 2009.

[4] T. Koch and A. Lapidoth, "On multipath fading channels at high SNR," IEEE Transactions on Information Theory, vol. 56, no. 12, pp. 5945-5957, 2010.

[5] A. Saad, M. Ismail, and N. Misran, "Rayleigh multiple input multiple," in Proceeding of the International Multi Conference of Engineers and Computer Scientists, vol. 2, 2009.

[6] A. Knopp, R. T. Schwarz, C. A. Hofmann, M. Chouayakh, and B. Lankl, "Measurements on the impact of sparse multipath components on the LOS MIMO channel capacity," in Proceedings of the 4th IEEE International Symposium on Wireless Communication Systems (ISWCS '07), pp. 55-60, October 2007.

[7] X. Cheng, C. X. Wang, H. Wang et al., "Cooperative MIMO channel modeling and multi-link spatial correlation properties," 
IEEE Journal on Selected Areas in Communications, vol. 30, no. 2, pp. 388-396, 2012.

[8] C.-X. Wang, X. Cheng, and D. I. Laurenson, "Vehicle-to-vehicle channel modeling and measurements: recent advances and future challenges," IEEE Communications Magazine, vol. 47, no. 11, pp. 96-103, 2009.

[9] X. Cheng, C.-X. Wang, D. I. Laurenson, S. Salous, and A. V. Vasilakos, "An adaptive geometry-based stochastic model for non-isotropic MIMO mobile-to-mobile channels," IEEE Transactions on Wireless Communications, vol. 8, no. 9, pp. 4824-4835, 2009.

[10] F. Perez Fontan and P. Marino Espineria, Modeling the Wireless Propagation Channel-A Simulation Approach With MATLAB, John Willey \& Sons, 2008.

[11] P. Kyosti et al., "WINNER II Channel Models," IST-WINNER D1.1.2v1.2, Sep. 2007.

[12] D. Tse and P. Viswanath, Fundamntal of Wireless Communication, Cabridge University Press, Cambridge, UK, 2005. 

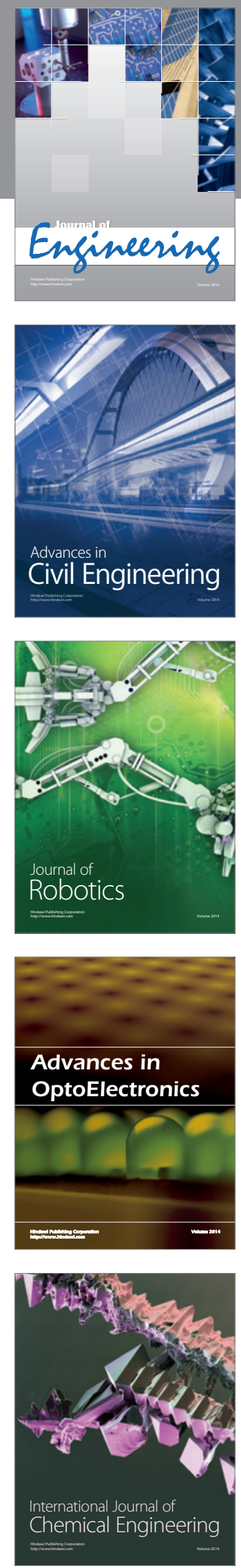

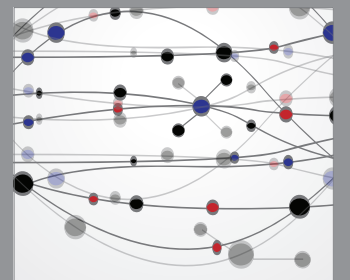

The Scientific World Journal
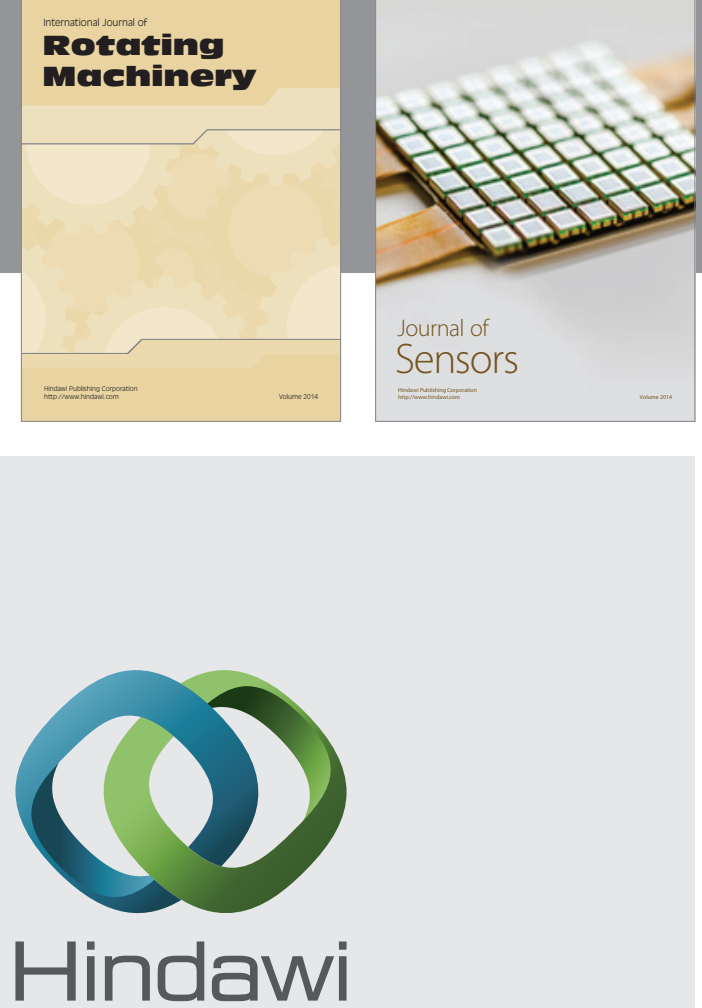

Submit your manuscripts at http://www.hindawi.com
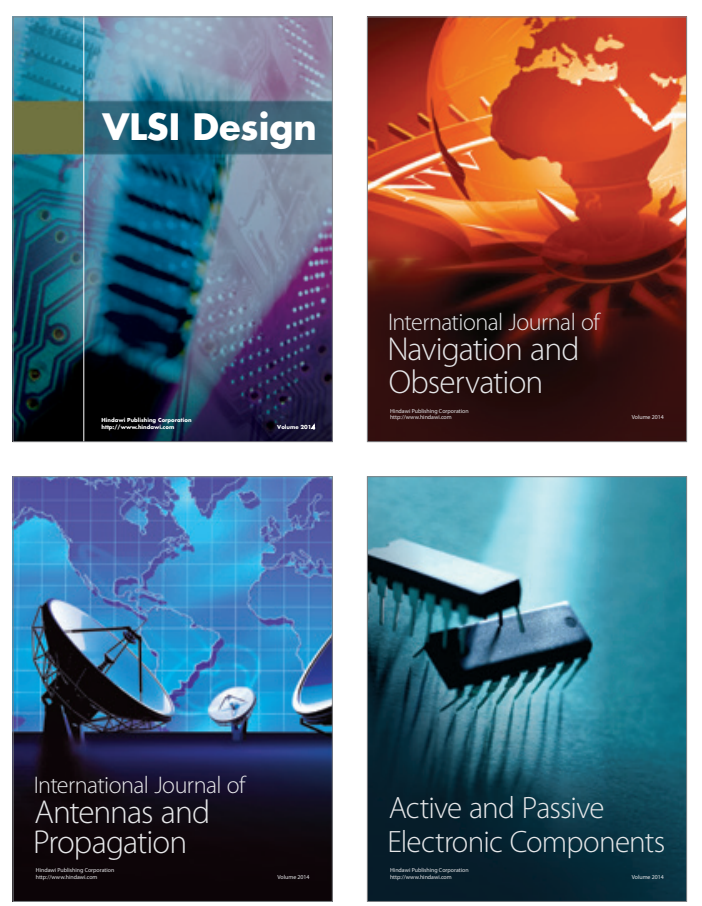
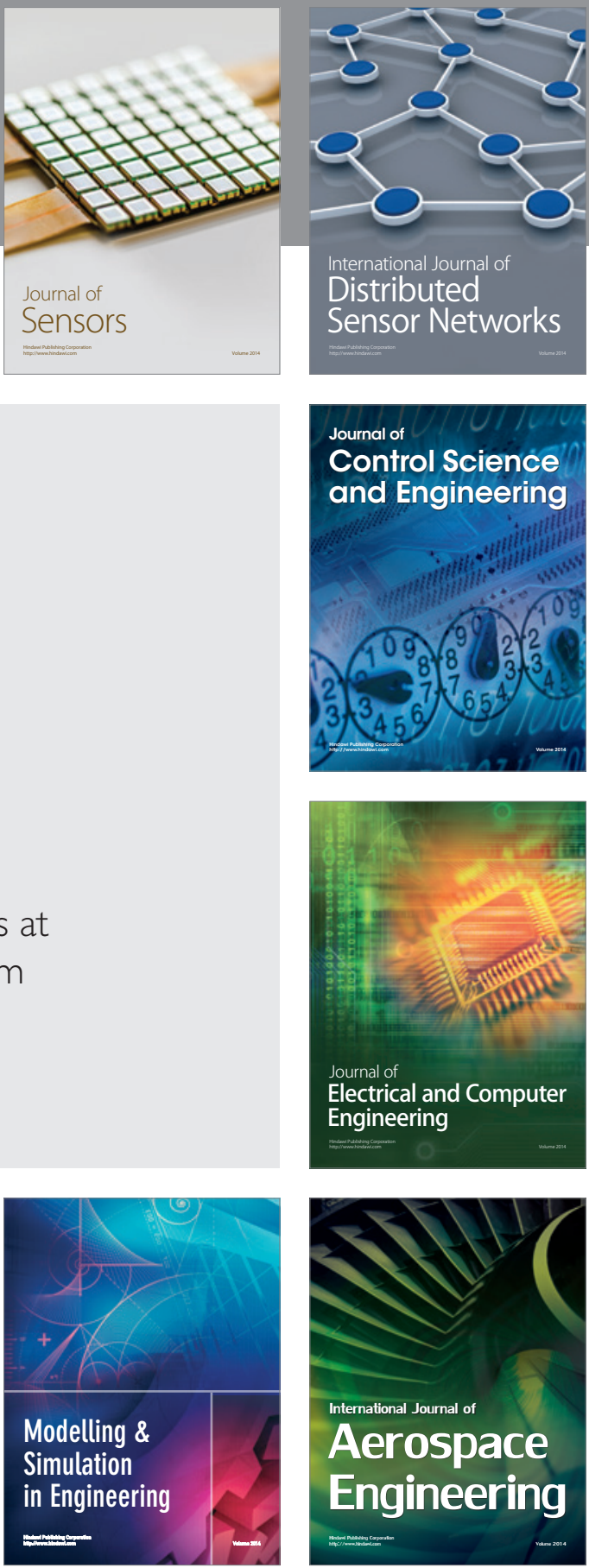

Journal of

Control Science

and Engineering
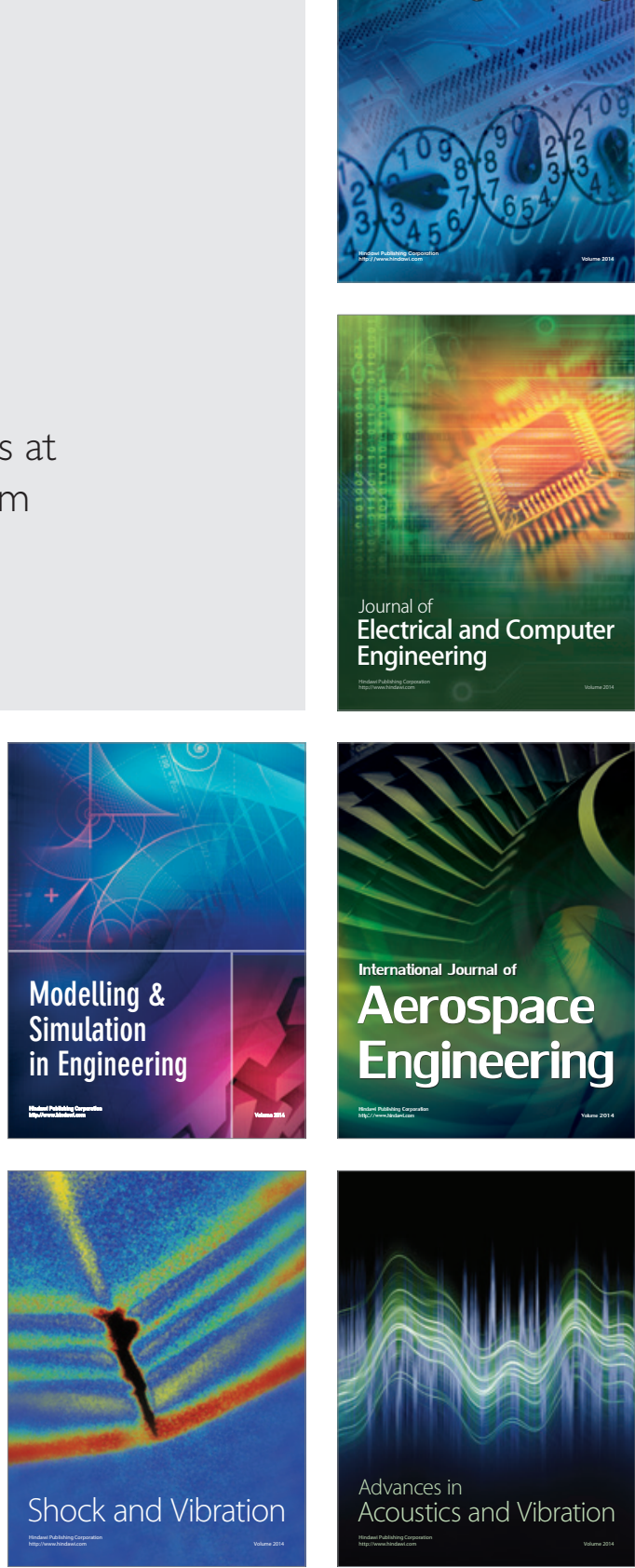